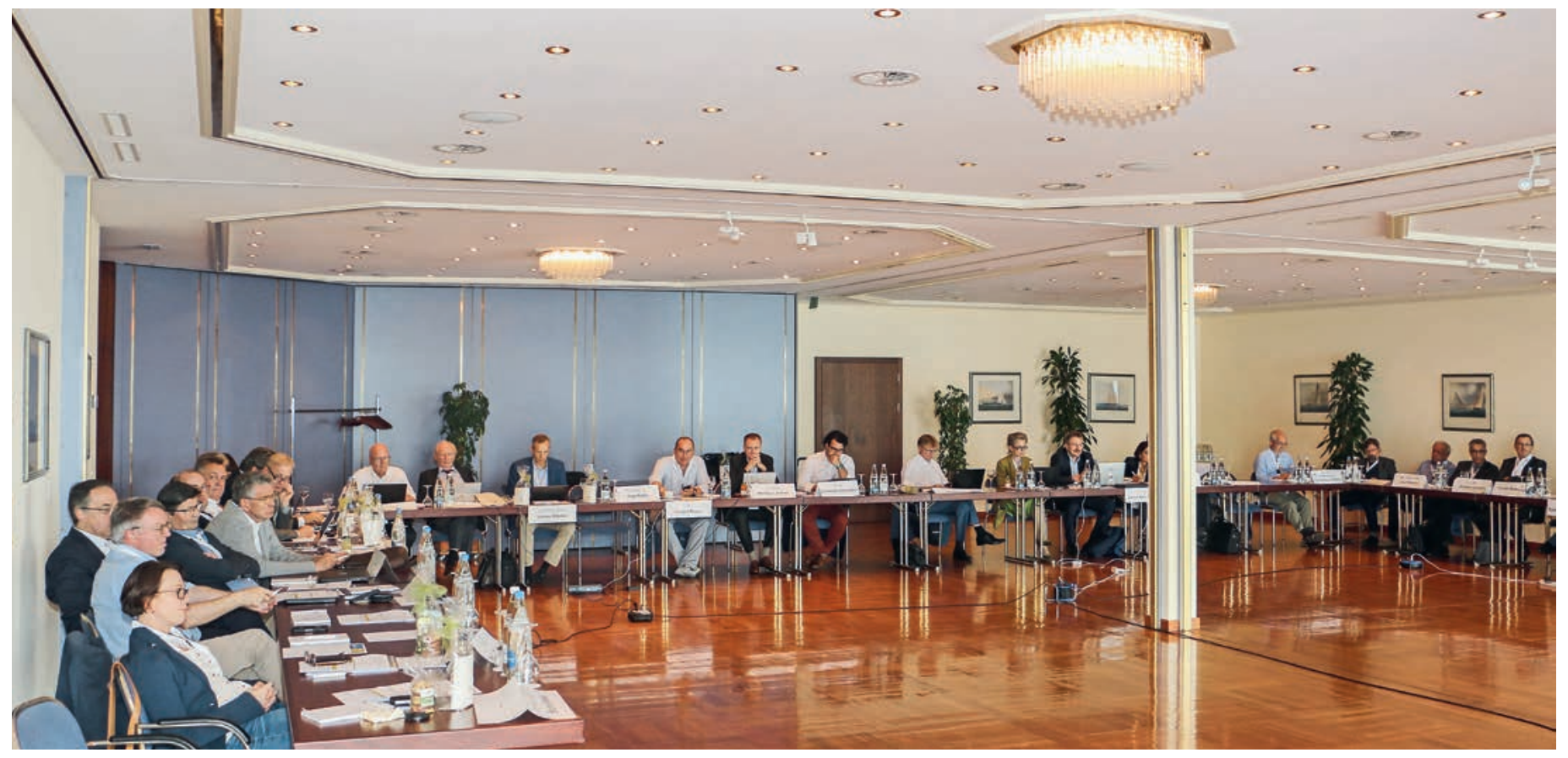

La Conférence consultative des sociétés médicales germanophones de 2018 a réuni des délégations d'Allemagne, d'Autriche, du Liechtenstein, du Luxembourg, de Belgique, de la Suisse et de la province italienne de Bolzano.

\title{
Des échanges intenses sur des sujets d'actualité
}

\section{Bruno Kesseli}

Dr méd. et lic. phil., rédacteur en chef

La FMH a accueilli la Conférence consultative des sociétés médicales germanophones de 2018, qui s'est tenue du 5 au 7 juillet à Horn, au bord du lac de Constance. Malgré ses 64 ans d'existence, cet événement devenu une tradition n'a pas pris une ride. Les défis auxquels les systèmes de santé des différents pays sont confrontés ont donné matière à discussion, comme le montre ci-après le compte rendu de la deuxième journée.

Inspiré par le ciel nuageux plombant le lac de Constance, le président de la FMH, Jürg Schlup, a ouvert la deuxième journée du symposium en comparant les conditions météorologiques ambiantes avec les scénarios parfois sombres de la politique de la santé auxquels sont confrontés les représentants des diverses sociétés médicales dans leurs pays. Jürg Schlup, qui a animé le programme de la matinée, s'est tout de même montré optimiste en évoquant les éclaircies annoncées pour l'après-midi...
La médecine, un processus basé sur la répartition des tâches sous responsabilité médicale

Après s'être consacrés la veille à la formation prégraduée, postgraduée et continue, les représentants des sociétés nationales se sont penchés sur le "perfectionnement des structures de soins» en mettant l'accent sur l'innovation, la concurrence et la sécurisation de la prise en charge. Quelques brèves présentations de pro- 


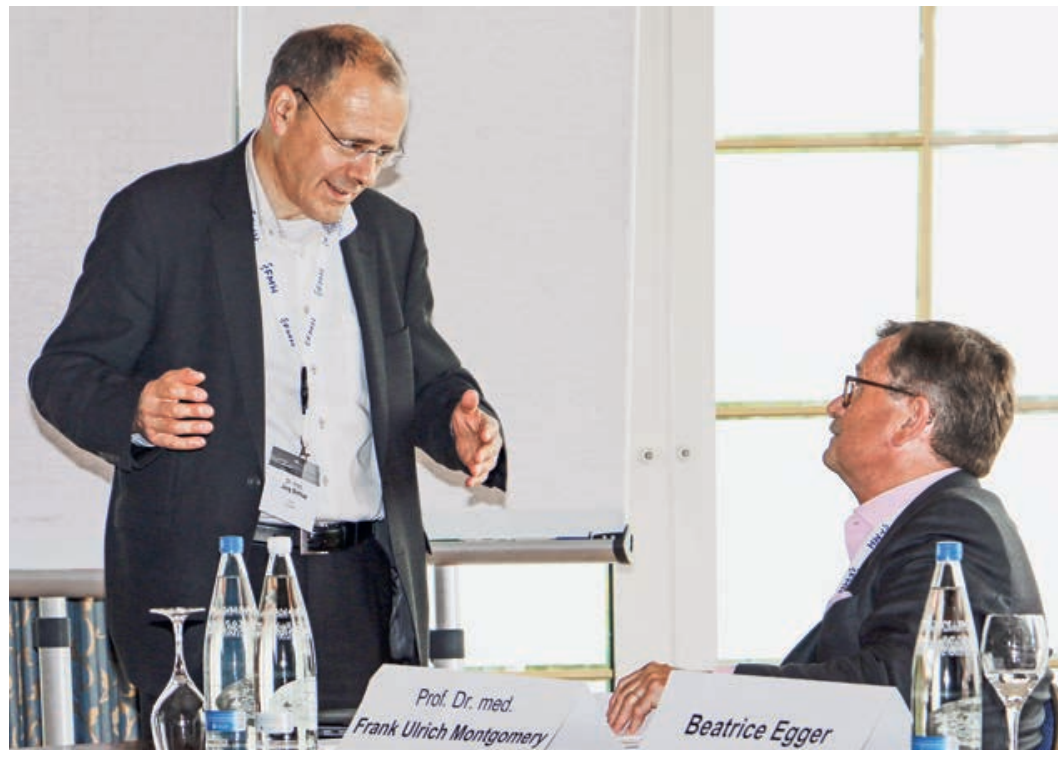

Le président de la FMH, Jürg Schlup, en conversation avec Frank Ulrich Montgomery, président de la Chambre médicale fédérale allemande.

jets de grande envergure en cours de réalisation ont révélé que l'Allemagne, l'Autriche et la Suisse font face à des défis comparables, bien que leurs systèmes de santé respectifs soient organisés de manière différente. La nécessité de rendre les soins plus efficaces s'impose cependant dans les trois pays, les facteurs économiques jouant un rôle central.

Harald Mayer, vice-président de la Fédération autrichienne des médecins salariés (Bundeskurie angestellter Ärzte), a présenté le programme «Médecin hospitalier 2025" mis sur pied par son association pour contribuer à améliorer les structures de soins dans le secteur hospitalier. Plaçant la relation médecin-patient

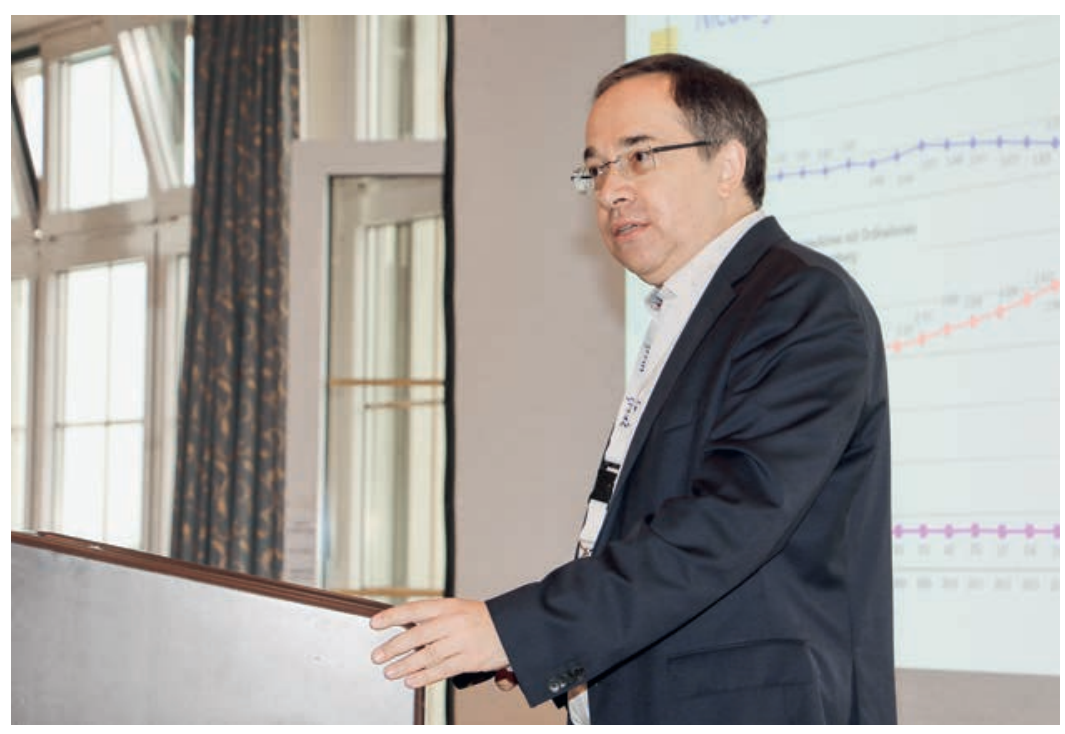

Johannes Zahrl, directeur de la Chambre médicale autrichienne, lors de sa présentation. et la sécurité des patients au cœur des préoccupations, ce programme conçoit la médecine comme un processus basé sur la répartition des tâches sous responsabilité médicale. Il prévoit une prise en charge échelonnée depuis le cabinet de groupe jusqu'à l'hôpital de référence, dont le personnel est disponible $24 \mathrm{~h}$ sur 24 , en passant par les unités de lits pour malades hospitalisés et les hôpitaux régionaux. Un système de rotation doit permettre aux médecins en formation de passer par les différentes étapes et un pool de spécialistes garantir des soins suffisants à tous les échelons. Différentes équipes d'experts sont regroupées pour former des unités médicales assurant la collaboration et la communication entre les spécialistes. Selon la fédération, le corps médical devrait en retirer un bénéfice au niveau des horaires, de la qualité des emplois, de l'équilibre entre vie professionnelle et vie privée ainsi que des perspectives professionnelles.

\section{Davantage de centres de soins primaires dirigés par des médecins de famille}

Johannes Zahrl, directeur de la Chambre médicale autrichienne (l'homologue de la FMH), a traité de la réorganisation des soins primaires dans son pays. Le «Programme pour des soins primaires multiprofessionnels et interdisciplinaires en Autriche», adopté par la Commission fédérale de contrôle des objectifs en 2014, prévoit tout particulièrement une bonne coordination de la prise en charge, notamment pour les malades chroniques, les enfants, les adolescents et les personnes âgées. Les heures d'ouverture des cabinets, de 7 à 19 h, devraient limiter les temps d'attente et augmenter le temps à disposition pour les discussions avec les patients, ce qui se traduirait par «un traitement médical et des conseils en santé globaux». Les principaux points positifs attendus pour le corps médical sont la collaboration entre les médecins et les autres professionnels de la santé, la réglementation des heures de travail pour un meilleur équilibre entre carrière et famille ainsi qu'une meilleure adéquation entre formation, recherche et pratique. Concrètement, les «nouveaux soins primaires» doivent être réalisés par la création d'«unités de soins primaires», c'est-à-dire de centres ambulatoires dans lesquels des généralistes assument la prise en charge de base des patients d'une région en collaborant avec des infirmiers, des physiothérapeutes, des assistants sociaux, des diététiciens, des ergothérapeutes et d'autres professionnels de la santé. Johannes Zahrl a cependant déploré que les fournisseurs de prestations n'aient pas été intégrés à la conception des projets. Selon lui, si les centres interprofessionnels de soins primaires sont une bonne idée 


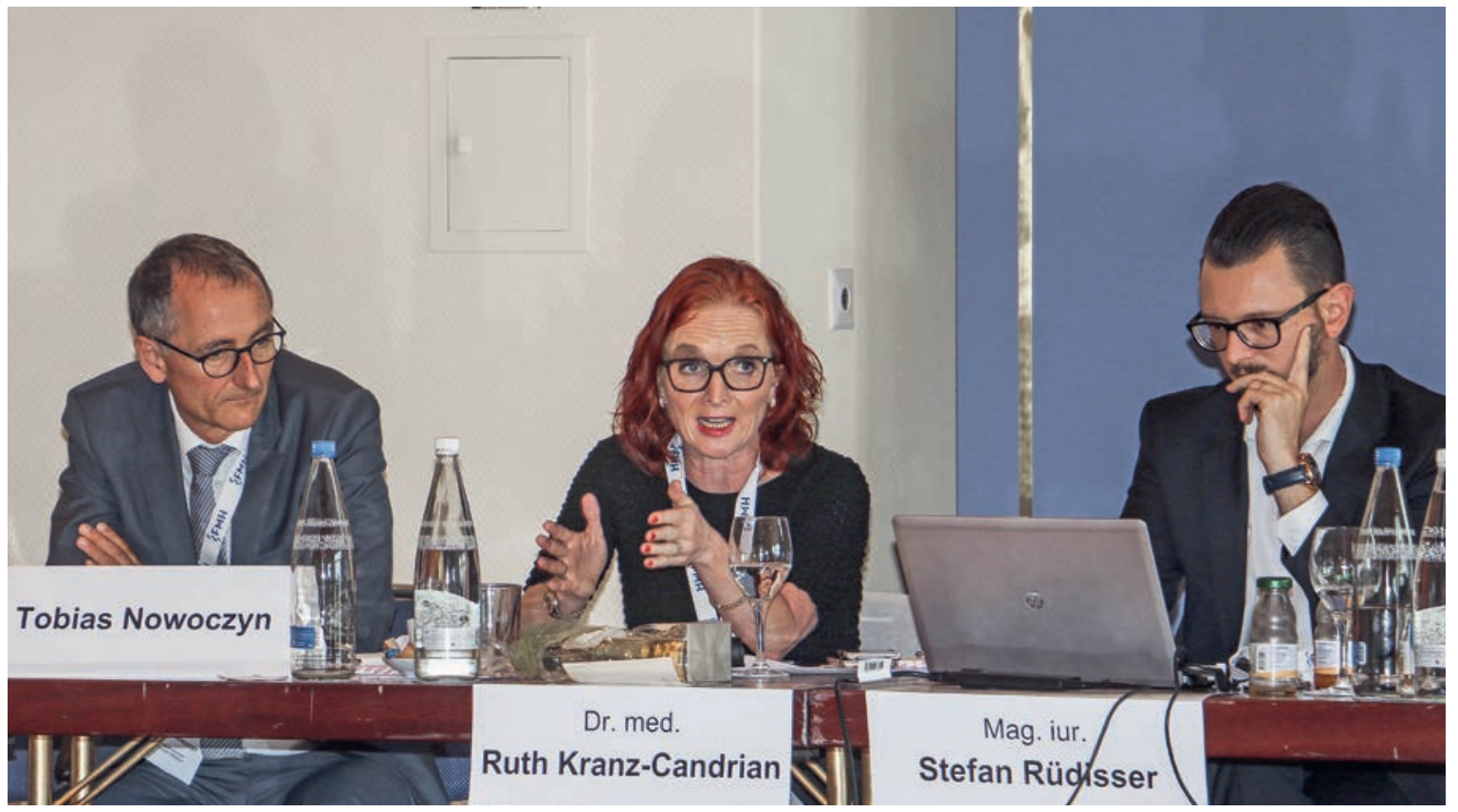

Ruth Kranz-Candrian, présidente de la Chambre médicale liechtensteinoise, en compagnie de son directeur Stefan Rüdisser et du directeur général de la Chambre médicale fédérale allemande, Tobias Nowoczyn.

à la base, le processus politique a créé trop de «mots vides de sens». Il ne reste plus qu’à attendre pour voir ce qu'il en sera réellement.

L'Allemagne suit les mêmes pistes, comme l'a montré la présentation de Max Kaplan, vice-président de la Chambre médicale fédérale allemande. La structure imaginée par le groupe de travail Médecine générale prévoit une "prise en charge collaborative» par des «équipes interprofessionnelles dirigées par un médecin de famille», garantissant des soins primaires intégrés intersectoriels et interprofessionnels. Il faut aussi revaloriser la profession d'employée médicale spécialisée (Medizinische Fachangestellte), l'équivalent de celle d'assistante médicale en Suisse. Tout comme en Autriche, on s'attend à ce que ce nouveau modèle apporte des avantages tant aux patients qu'aux fournisseurs de prestations. Grâce aux «centres dirigés par un médecin de famille», les patients devraient bénéficier de soins plus efficaces puisque mieux coordonnés, d'heures d'ouverture qui leur conviennent, de structures claires et d'un large éventail de services en un seul et même lieu. Les fournisseurs de prestations obtiendraient pour leur part des conditions plus attrayantes grâce à des horaires flexibles leur permettant de mieux concilier travail et vie privée, la possibilité de se décharger de certaines tâches et de meilleures opportunités d'échanges intra- et interprofessionnels. La réponse de Max Kaplan à la question de Jürg Schlup («Ces centres seront-ils réellement dirigés par des médecins et non par des gestionnaires?») ne pouvait pas être plus claire: "par des médecins, uniquement par des médecins", même si, il ne faut pas le cacher, les directeurs médicaux passeront une grande partie de leur temps de travail (env. 80\% selon les estimations) à accomplir des tâches administratives. D’après Max

\section{$64^{\mathrm{e}}$ Conférence consultative des \\ sociétés médicales germanophones}

La conférence de 2018 a réuni des délégations d'Allemagne, d'Autriche, du Liechtenstein, du Luxembourg, de Belgique, de la Suisse et de la province italienne de Bolzano. Cet événement accueilli par la FMH s'est tenu du 5 au 7 juillet à Horn, au bord du lac de Constance.

La première journée, dirigée par Werner Bauer, président de I'Institut suisse pour la formation médicale postgraduée et continue (ISFM), a été consacrée à la formation. Le lendemain, les présentations et discussions conduites par Jürg Schlup, président de la FMH, et Frank Ulrich Montgomery, président de la Chambre médicale fédérale allemande, se sont concentrées sur les sujets "Développement des structures de soins et innovation» et "Evolution de l'image du médecin». Que fera le médecin à I'avenir ou que fera le médecin de demain? La troisième journée a commencé par l'exposé d'une intervenante externe, Agnes Bäker, qui a tenté de répondre à la question «Pourquoi les meilleurs hôpitaux sont-ils dirigés par des médecins?» [1]. Les présidents et délégués des organisations faîtières ont ensuite parlé de la situation dans leurs pays en abordant des sujets d'actualité importants pour la profession médicale en Allemagne (Frank Ulrich Montgomery), en Autriche (Thomas Szekeres), au Liechtenstein (Ruth Kranz-Candrian), au Luxembourg (Alain Schmit), à Bolzano (Monica Oberrauch), en Belgique (Michael Heinen) et en Suisse (Jürg Schlup). 


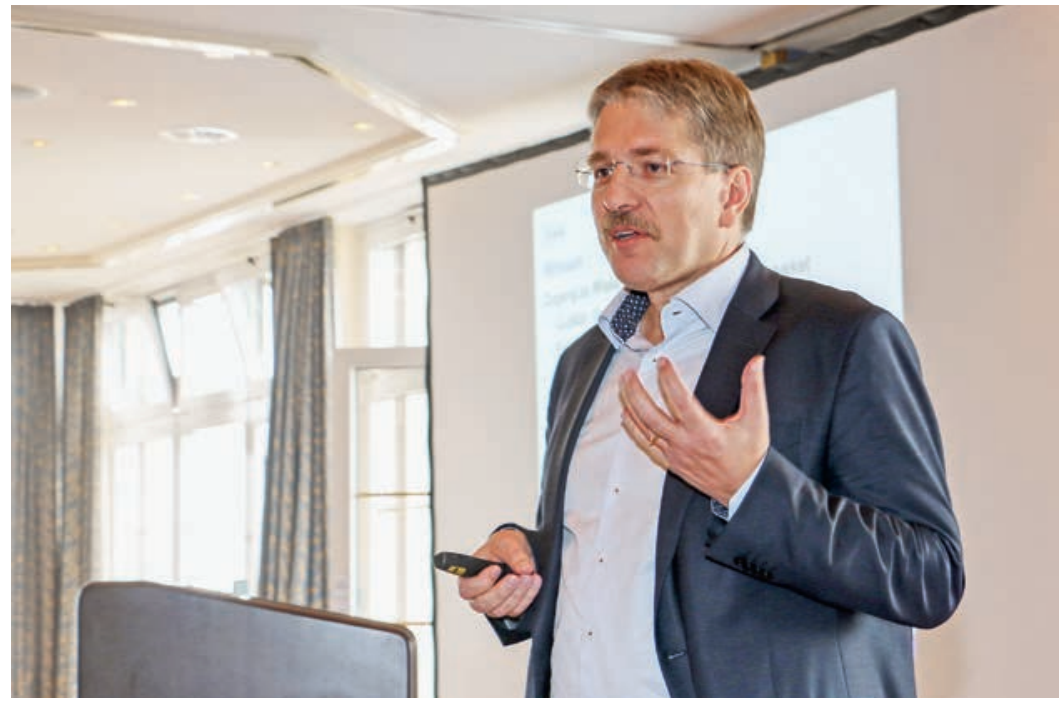

Lors de sa présentation, le Dr Carlos Quinto, membre du Comité central de la FMH, s'est concentré sur la relation d'égal à égal avec le patient.

Kaplan, ce type de centres amélioreront également les soins dans les régions rurales et celles aux structures peu développées.

\section{Importance incontestée de la collabora- tion interprofessionnelle}

Barbara Weil, cheffe de la division Santé publique et professions de la santé à la FMH, a montré comment la collaboration interprofessionnelle, une démarche souvent qualifiée de prometteuse, se concrétise dans le domaine de la prévention. Selon Barbara Weil, si tout le monde reconnaît l'importance de la prévention dans les soins de santé d'une population, les médecins en exercice se voient confrontés à différentes activités et attentes. Dans ce contexte, la coordination, un sens de la qualité partagé et des conditions-cadres communes s'avèrent cruciaux. En Suisse, la collaboration interprofessionnelle et coordonnée a déjà permis le développement d'un certain nombre d'outils favorisant une prévention et une promotion de la santé globales, systématiques et dont l'efficacité a pu être démontrée. La base de données générique «EviPrev» (www.eviprev.ch), l'entretien motivationnel en tant que forme de communication générique, des modules sur les facteurs de risque, le coaching santé ou encore des outils pour la prise de décision et la planification partagées n'en sont que quelques exemples.

\section{Choisir le changement au lieu de le subir}

Le fait que la coopération interprofessionnelle deviendra de plus en plus importante - et à juste titre - a été souligné par plusieurs intervenants lors de la discussion qui a suivi les exposés du matin. Günther Jonitz l'a qualifiée de principe de base et a assuré que les médecins indépendants sont les plus efficaces en ce qui concerne l'organisation des soins primaires. D'autres participants ont objecté que les médecins de la génération actuelle ne sont plus prêts à prendre des risques (financiers) et préfèrent être employés. Le président de la Chambre médicale fédérale allemande, Frank Ulrich Montgomery, a précisé que des modèles à risque modéré sont également prévus. Les médecins pourraient acquérir une expérience sans risque dans un «cabinet de stage» pendant trois à cinq ans, puis reprendre le cabinet si cette forme de soins primaires leur convient. En fin de matinée, Günther Jonitz, président de la Chambre médicale de Berlin, a abordé les «défis pour la profession médicale». A l'aide de nombreux exemples frappants et avec beaucoup d'humour, il a montré comment les médecins ont vu leur rôle évoluer au cours des dernières années, passant du statut de «seuls responsables» à celui de "responsables ultimes», alors que l'«autodétermination» était remplacée progressivement par l'«hétéronomie». Mais loin de se plaindre de dysfonctionnements et de regretter le passé, Günther Jonitz estime qu'une professionnalisation accrue du corps médical constitue la meilleure solution. Il encourage les médecins à prendre leur responsabilité et à conduire eux-mêmes le changement. Les compétences médicales devraient être renforcées et les qualifications étendues. En fin de compte, c'est aux médecins de décider s'ils préfèrent choisir ou subir le changement. Son exposé s'est terminé sur ces mots: «Ce sont ceux qui défendent les soins aux patients de façon convaincante qui ont le dernier mot dans la discussion sur la politique de santé.»

\section{L'image du médecin en mutation}

Les intervenants de l'après-midi ont repris plusieurs des défis abordés par Günther Jonitz. Intitulé «Bureaucratisation, codage, administration et gestion de la qualité: reste-t-il du temps pour l'activité médicale?», l'exposé de Jörg Hutter, vice-président de la Chambre médicale de Salzbourg, a traité d'un sujet également bien débattu en Suisse et en Allemagne. Si les systèmes de dossiers médicaux électroniques promettent des gains d'efficacité et une amélioration de la qualité et de la sécurité du traitement pour les patients, ils n'entraînent aucune réduction de la charge administrative pour le personnel médical. Selon Jörg Hutter, il ne suffit pas de déléguer des tâches administratives à d'autres groupes professionnels, dont certains sont même spécialisés dans la documentation médicale, il faut aussi 


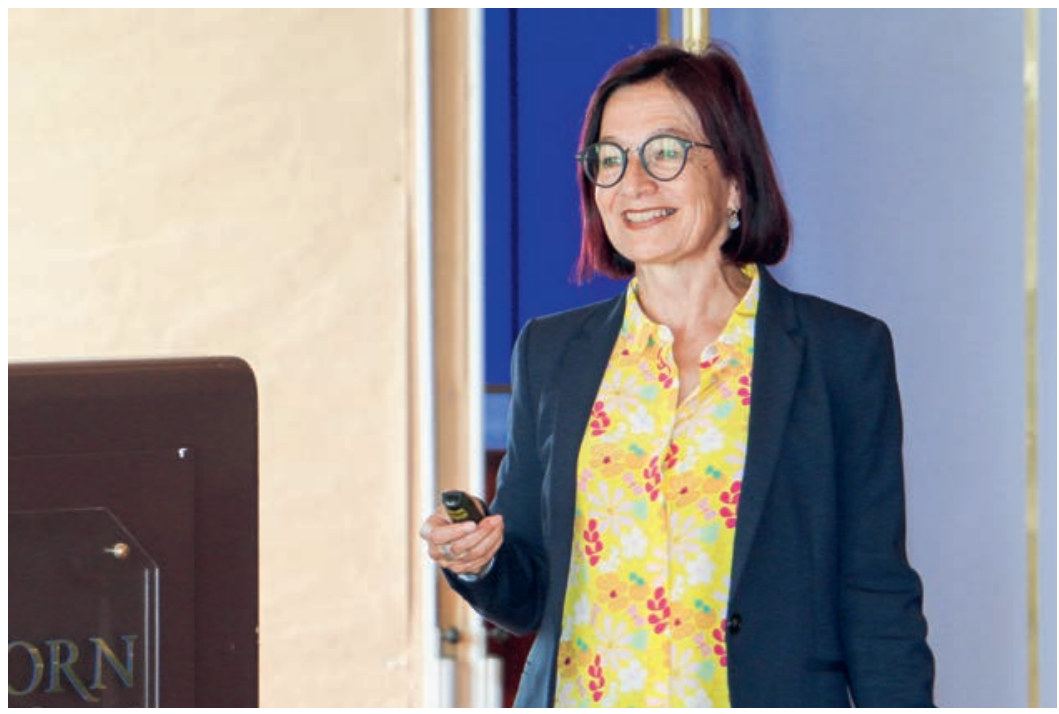

Yvonne Gilli, membre du Comité central de la FMH, s'est exprimée sur la numérisation.

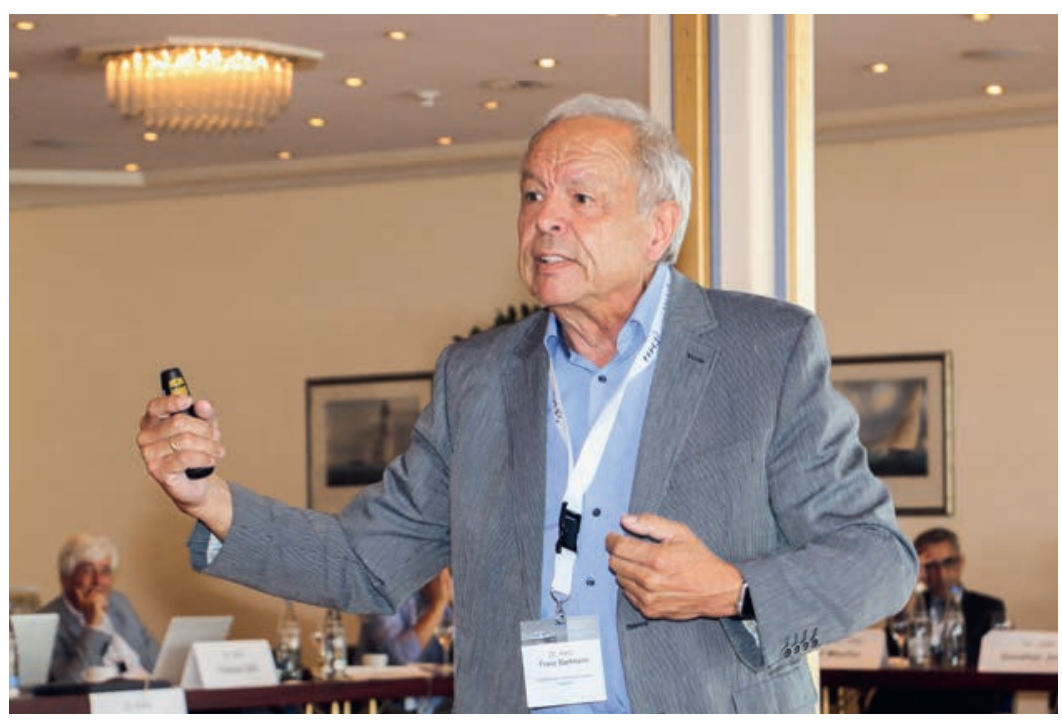

Franz Joseph Bartmann, président de la Chambre médicale du Schleswig-Holstein, a parlé avec ferveur des nouvelles structures de soins résultant de la numérisation.

adapter la formation des médecins-assistants aux nouvelles possibilités numériques. Il est encore difficile d'évaluer à quel point les différentes mesures actuellement à l'étude permettront réellement d'améliorer la situation.

Carlos Quinto, membre du Comité central de la FMH, s'est concentré sur la relation d'égal à égal avec le patient. Si selon lui la prise de décision partagée et l'autonomisation sont des éléments importants de soins médicaux centrés sur le patient au sens de la Charte d'Ottawa pour la promotion de la santé, il est impossible d'éluder une certaine asymétrie dans la relation médecin-patient surtout, paradoxalement, si les patients sont pris au sérieux. Les décisions responsables prises conjointement par le patient et le médecin doivent en tenir compte.

\section{Le smartphone est-il le stéthoscope du $\mathrm{XXI}^{\mathrm{e}}$ siècle?}

La fin de l'après-midi a été consacrée à l'influence de la numérisation sur le rôle des médecins et sur la relation médecin-patient. Yvonne Gilli, membre du Comité central de la FMH, Josef Mischo, président de la Chambre médicale de la Sarre, et Franz Joseph Bartmann, président de la Chambre médicale du Schleswig-Holstein, ont convenu que le problème n'était pas la numérisation, mais sa mise en œuvre. Yvonne Gilli a souligné, par exemple, que la numérisation ne comble pas l'écart entre les régions riches et pauvres du monde et qu'en soi elle n'augmente pas la productivité. Franz Joseph Bartmann a observé que la médecine était en retard par rapport à d'autres domaines - comme l'industrie musicale - en termes d'exploitation des possibilités numériques, tout en restant convaincu que le smartphone deviendra le "stéthoscope du XXI ${ }^{e}$ siècle» grâce aux progrès de la télémédecine. La majorité des participants ont approuvé sa conclusion: les applications ne remplaceront pas les médecins, mais la numérisation changera fondamentalement le rôle des médecins.

\section{Crédit photos}

Photos: Bruno Kessel

\section{Référence}

1 Voir aussi à ce sujet l'entretien publié dans le Bulletin des médecins suisses du 8 novembre 2017 (disponible en ligne): Kesseli B. «L'évidence plaide pour des médecins aux postes de direction.» Entretien avec Agnes Bäker, professeure assistante en management des organisations à but non lucratif à l'Université de Zurich. Bulletin des médecins suisses. 2017;98(45):1487-9. DOI: https://doi.org/10.4414/saez.2017.06129 